\title{
Herbrand consistency and bounded arithmetic
}

\author{
by
}

\author{
Zofia Adamowicz (Warszawa)
}

\begin{abstract}
We prove that the Gödel incompleteness theorem holds for a weak arithmetic $T_{m}=I \Delta_{0}+\Omega_{m}$, for $m \geq 2$, in the form $T_{m} \forall \forall \operatorname{HCons}\left(T_{m}\right)$, where $\operatorname{HCons}\left(T_{m}\right)$ is an arithmetic formula expressing the consistency of $T_{m}$ with respect to the Herbrand notion of provability. Moreover, we prove $T_{m} \not$ HCons $^{I_{m}}\left(T_{m}\right)$, where HCons ${ }^{I_{m}}$ is HCons relativised to the definable cut $I_{m}$ of $(m-2)$-times iterated logarithms. The proof is model-theoretic. We also prove a certain non-conservation result for $T_{m}$.
\end{abstract}

In $[\mathrm{PW}]$ Paris and Wilkie asked the following question: does $I \Delta_{0}$ prove the cut free consistency of $I \Delta_{0}$ ? Here we solve (negatively) an analogous question with $I \Delta_{0}+\Omega_{m}, m \geq 2$, in place of $I \Delta_{0}$. The theory $I \Delta_{0}+\Omega_{m}$ can be considered as another version of bounded arithmetic and Herbrand provability is a version of cut free provability (it is defined and formalized in Section 2).

Pudlák [P] and Hájek-Pudlák [HP] proved Gödel's Incompleteness Theorem for weak arithmetic with the ordinary (Hilbert) notion of provability.

As Herbrand consistency of a theory is a weaker statement than its ordinary consistency, proving its unprovability in some theory is more difficult.

In $[\mathrm{P}]$ Pudlák also proves that theories of the form $I \Delta_{0}+\Omega_{m}$ do prove their own Herbrand consistency relativised to a certain definable cut $J_{m}$. Here we show that they do not prove their own Herbrand consistency relativised to $I_{m}$. It follows that consistently $J_{m} \subsetneq I_{m}$.

Pudlák $[\mathrm{P}]$ (see also Hájek and Pudlák [HP]) in his proof uses a provability predicate Prov and its restriction Prov* to a definable initial segment and shows that Prov and Prov* satisfy some derivability conditions from which the main result is obtained in a routine way.

Our result for the case of $I \Delta_{0}+\Omega_{2}(m=2)$ has been proved in [AZ] and for $m=1$ in [A1]. In [AZ] we applied an idea similar to that of [P] and [HP].

2000 Mathematics Subject Classification: Primary 03F30.

Research supported in part by The State Committee for Scientific Research (Poland), KBN, grant number 5 P03A-037 20. 
In this paper we give a different proof, much more model-theoretic than the former one.

The Paris-Wilkie problem has also been considered by Willard [W].

We use standard notation throughout. In particular, $\Delta_{0}$ denotes the class of bounded arithmetical formulas and $I \Delta_{0}$ is the system of weak arithmetic with induction scheme for $\Delta_{0}$ formulas only. $B \Sigma_{1}$ denotes the $\Sigma_{1}$ collection scheme. Addition and multiplication are regarded as relations.

Let $\omega_{0}(x)=x^{2}$ and $\omega_{m+1}(x)=2^{\omega_{m}(\log x)}$ (arithmetical log denotes the integral part of the logarithm). The axiom $\Omega_{m}$ states the totality of the function $\omega_{m}$. The axiom exp states the totality of the exponential function $y=2^{x}$.

Generally formulas are always defined as elements of $\mathbb{N}$ or of a model $M$ under consideration. In other words we identify formulas with their Gödel numbers.

Let Sat be a universal formula for $\Delta_{0}$. Thus Sat is $\Sigma_{1}$ and

$$
M \models \operatorname{Sat}(\varphi) \quad \text { iff } \quad M \models \varphi,
$$

for $\varphi \in \Delta_{0}$, in every model $M$ of $I \Delta_{0}+\exp$.

For each $n \in \mathbb{N}$ let

$$
\log ^{n} M=\left\{a \in M: \exists b \in M\left(M \models\left(b=\exp ^{n}(a)\right)\right)\right\} .
$$

Of course every $\log ^{n} M$ is a definable initial segment of $M$ (" $y=\exp (x)$ " can be expressed by a $\Delta_{0}$ formula- see $\left.[\mathrm{HP}]\right)$. Thus we have $I_{m}^{M}=\log ^{m-2} M$.

1. We shall express (in Sec. 2) the Herbrand consistency by a $\Pi_{1}$ formula $\operatorname{HCons}_{m}(\varphi)\left(\varphi\right.$ is Herbrand consistent with $T_{m}$ ). We shall also use an auxiliary $\Pi_{1}$ formula HCons $I_{m}^{I_{m}}(\varphi)$, obtained from HCons Hen $_{m}$ by restriction of the initial quantifier to the definable segment $I_{m}$ (in the standard model $I_{m}$ is $\mathbb{N}$ ). The formula $\mathrm{HCons}_{m}^{I_{m}}$ will have the following property:

(*) For a bounded $\theta$ if

$$
T_{m}+\exists \bar{x} \in \log ^{m+1} \theta(\bar{x})+\operatorname{HCons}_{m}^{I_{m}}(" 0=0 ")
$$

is consistent then so is

$$
T_{m}+\exists \bar{x} \in \log ^{m+2} \theta(\bar{x}) .
$$

Note that HCons H $_{m}$ ( $0=0 "$ ") expresses " $T_{m}$ is Herbrand consistent".

Now with HCons ${ }_{m}$ and HCons ${ }_{m}^{I_{m}}$ as above we can prove the announced result,

$$
T_{m} \nvdash \operatorname{HCons}_{m}^{I_{m}}(" 0=0 ") .
$$

We need the following theorem: 
1.1. Theorem. For $m, n \in \mathbb{N}$ there is a bounded formula $\theta(\bar{x})$ (where $\bar{x}$ is a finite string of variables) such that

$$
I \Delta_{0}+\Omega_{n}+\exists \bar{x} \in \log ^{m} \theta(\bar{x})
$$

is consistent and

$$
I \Delta_{0}+\Omega_{n}+\exists \bar{x} \in \log ^{m+1} \theta(\bar{x})
$$

is inconsistent.

In particular, for $m \in \mathbb{N}$ there is a bounded formula $\theta_{m}(\bar{x})$ such that

$$
I \Delta_{0}+\Omega_{m}+\exists \bar{x} \in \log ^{m+1} \theta_{m}(\bar{x})
$$

is consistent and

$$
I \Delta_{0}+\Omega_{m}+\exists \bar{x} \in \log ^{m+2} \theta_{m}(\bar{x})
$$

is inconsistent.

The theorem can be considered as a certain non-conservation result and may be interesting in its own right. We prove it later in this section.

Now the proof of the main result is as follows. Let $\theta_{m}$ be given by Theorem 1.1. We shall show that

$$
T_{m}+\exists \bar{x} \in \log ^{m+1} \theta_{m}(\bar{x})+\operatorname{HCons}_{m}^{I_{m}}(" 0=0 ")
$$

is inconsistent.

Suppose that this theory is consistent. Then, by $(*)$,

$$
T_{m}+\exists \bar{x} \in \log ^{m+2} \theta_{m}(\bar{x})
$$

is consistent. But this violates the choice of $\theta_{m}$. Hence

$$
\left.T_{m}+\exists \bar{x} \in \log ^{m+1} \theta_{m}(\bar{x})+\operatorname{HCons}_{m}^{I_{m}} \text { (" } 0=0 "\right)
$$

is inconsistent. Thus, in view of the consistency of $T_{m}+\exists \bar{x} \in \log ^{m+1} \theta_{m}(\bar{x})$, we have

$$
T_{m} \not \text { HCons }_{m}^{I_{m}}(" 0=0 "),
$$

which completes the proof.

We have shown that to prove our main result it is sufficient to construct formulas $\mathrm{HCons}_{m}, \mathrm{HCons}_{m}^{I_{m}}$ with the properties stated above. This will be done in subsequent sections.

Now let us prove Theorem 1.1.

Let $m, n \in \mathbb{N}$. Suppose that for every bounded formula $\theta$ such that $I \Delta_{0}+\Omega_{n}+\exists \bar{x} \in \log ^{m} \theta(\bar{x})$ is consistent, $I \Delta_{0}+\Omega_{n}+\exists \bar{x} \in \log ^{m+1} \theta(\bar{x})$ is consistent. Fix a bounded formula $\theta_{0}$ such that

$$
I \Delta_{0}+\Omega_{n}+\exists \bar{x} \in \log ^{m} \theta_{0}(\bar{x})
$$

is consistent, $\bar{x}=x_{1}, \ldots, x_{k}$. Hence

$$
I \Delta_{0}+\Omega_{n}+\exists \bar{x} \in \log ^{m+1} \theta_{0}(\bar{x})
$$


is consistent. Therefore

$$
I \Delta_{0}+\Omega_{n}+\exists y \in \log ^{m} \exists \bar{x} \leq y\left(\bigwedge_{i=1, \ldots, k} y \geq 2^{x_{i}} \wedge \theta_{0}(\bar{x})\right)
$$

is consistent.

Let $\theta_{1}(y)$ be $\exists \bar{x} \leq y\left(\bigwedge_{i=1, \ldots, k} y \geq 2^{x_{i}} \wedge \theta_{0}(\bar{x})\right)$. Applying our supposition to $\theta_{1}$ we infer

$$
I \Delta_{0}+\Omega_{n}+\exists y \in \log ^{m+1} \exists \bar{x} \leq y\left(\bigwedge_{i=1, \ldots, k} y \geq 2^{x_{i}} \wedge \theta_{0}(\bar{x})\right)
$$

is consistent. Hence

$$
I \Delta_{0}+\Omega_{n}+\exists \bar{x} \in \log ^{m+2} \theta_{0}(\bar{x})
$$

is consistent. Continuing we infer that

$$
I \Delta_{0}+\Omega_{n}+\exists \bar{x} \in \log ^{m+n^{\prime}} \quad \theta_{0}(\bar{x})
$$

is consistent for all $n^{\prime} \in \mathbb{N}$. Thus there is a model $M$ of $I \Delta_{0}$ and an $\bar{a} \in M$ such that $M=\theta_{0}(\bar{a})$ and $M=\left(\exp ^{n^{\prime}}(\max \bar{a})\right.$ exists) for $n^{\prime} \in \mathbb{N}$. Consider the initial segment $M^{\prime}$ of $M$ determined by the elements $\exp ^{n^{\prime}}(\max \bar{a})$ for $n^{\prime} \in \mathbb{N}$. Then $M^{\prime} \models I \Delta_{0}+\exp$ and $M^{\prime}=\theta_{0}(\bar{a})$. It follows that the theory $I \Delta_{0}+\exp +\exists \bar{x} \theta_{0}(\bar{x})$ is consistent.

Thus, for every bounded $\theta$, if $I \Delta_{0}+\Omega_{n}+\exists \bar{x} \in \log ^{m} \theta(\bar{x})$ is consistent then so is $I \Delta_{0}+\exp +\exists \bar{x} \theta(\bar{x})$.

Also, for any bounded $\theta_{1}, \ldots, \theta_{l}$, if

$$
I \Delta_{0}+\Omega_{n}+\bigwedge_{i=1, \ldots, l} \exists \bar{x} \in \log ^{m} \theta_{i}(\bar{x})
$$

is consistent then so is

$$
I \Delta_{0}+\exp +\bigwedge_{i=1, \ldots, l} \exists \bar{x} \theta_{i}(\bar{x})
$$

because the sentence $\bigwedge_{i=1, \ldots, l} \exists \bar{x} \theta_{i}(\bar{x})$ can be presented as

$$
\exists \bar{x}_{1}, \ldots, \bar{x}_{l} \bigwedge_{i=1, \ldots, l} \theta_{i}\left(\bar{x}_{i}\right) .
$$

Let $\Sigma_{1}^{*}$ denote the collection of all sentences of the form $\exists \bar{x} \in \log ^{m} \theta(\bar{x})$, where $\theta$ is bounded. Let $T^{*} \subseteq \Sigma_{1}^{*}$ be maximal (with respect to $\Sigma_{1}^{*}$ ) consistent with $I \Delta_{0}+\Omega_{n}$. It follows that $I \Delta_{0}+\exp +T^{*}$ is consistent.

Let $T^{* *} \subseteq \Sigma_{1}$ consist of those $\Sigma_{1}$ sentences $\phi$ of the form $\exists \bar{x} \theta(\bar{x})$, where $\theta$ is bounded, for which the sentence " $\exists \bar{x} \in \log ^{m} \theta(\bar{x})$ " is in $T^{*}$. We shall show that $T^{* *}$ is maximal consisting of $\Sigma_{1}$ sentences consistent with $I \Delta_{0}+\exp$. 
Let $\phi \in \Sigma_{1}$ of the form $\exists \bar{x} \theta(\bar{x})$ be such that $I \Delta_{0}+\exp +T^{* *}+\phi$ is consistent. Then

$$
I \Delta_{0}+\exp +T^{* *}+\exists \bar{x} \in \log ^{m} \theta(\bar{x})
$$

is consistent. Hence

$$
I \Delta_{0}+\Omega_{n}+T^{*}+\exists \bar{x} \in \log ^{m} \theta(\bar{x})
$$

is consistent. Hence, by the maximality of $T^{*}$, the sentence " $\exists \bar{x} \in \log ^{m} \theta(\bar{x})$ " is in $T^{*}$, whence $\phi \in T^{* *}$. It follows that $T^{* *}$ is maximal consisting of $\Sigma_{1}$ sentences consistent with $I \Delta_{0}+\exp$.

Let $M=I \Delta_{0}+\exp +T^{* *}+B \Sigma_{1}$ be such that $\Sigma_{1}(M)$ (the set of $\Sigma_{1}$ sentences true in $M$ ) is not coded in $M$. Such a model $M$ exists (see [WP2], the proof of Theorem 9$)$. Note that by the maximality of $T^{* *}, \Sigma_{1}(M)=T^{* *}$.

By another result of [WP2] (Theorem 5(2)), $M$ has a proper end-extension to a model $M^{\prime}$ of $I \Delta_{0}+\Omega_{n+1}$. By the maximality of $T^{*}$ with respect to $I \Delta_{0}+\Omega_{n}$ and $\Sigma_{1}^{*}$, we have

$$
M^{\prime} \models \phi \Leftrightarrow M \models \phi,
$$

for every $\phi \in \Sigma_{1}^{*}$. Let $a \in M^{\prime} \backslash M$. We thus have

$$
M \models \phi \Leftrightarrow M^{\prime} \models \phi^{a},
$$

for every $\phi \in \Sigma_{1}^{*}$.

Since every $\phi \in \Sigma_{1}$ is equivalent in $M$ (and so in $M^{\prime}$ ) in a canonical way to an $\exists \Sigma_{1}^{b}$ sentence (via the Matiyasevich theorem) and $M^{\prime} \models \Omega_{1}$, we may use the universal formula for $\exists \Sigma_{1}^{b}$ formulas available in $M^{\prime}$ to infer that

$$
\left\{\phi \in \Sigma_{1}^{*}: M^{\prime} \models \phi^{a}\right\}
$$

is coded in $M^{\prime}$. Here $\Sigma_{1}^{b}$ denotes Buss's class (see [B], [HP]) and $\exists \Sigma_{1}^{b}$ denotes the class of formulas of the form $\exists \bar{x} \theta(\bar{x})$, where $\theta$ is $\Sigma_{1}^{b}$. The required universal formula can be built using the formula $\mu_{1}$ from Theorem 4.18 of $[\mathrm{HP}]$ (see also the appendix of $[\mathrm{A}]$ ). The notation $\phi^{a}$ denotes the formula obtained from $\phi$ by bounding its unbounded existential quantifiers to $a$.

But then $T^{*}$ is coded in $M^{\prime}$ and consequently so is $T^{* *}$; hence $\Sigma_{1}(M)$ is coded in $M^{\prime}$, whence it is coded in $M$, contradiction.

Thus the theorem has been proved.

2. Let us recall what we mean by Herbrand type provability of a sentence. Let $\varphi$ be a sentence of the form

$$
\exists x_{1} \forall y_{1} \ldots \exists x_{m} \forall y_{m} \bar{\varphi}\left(x_{1}, y_{1}, \ldots, x_{m}, y_{m}\right),
$$

where $\bar{\varphi}$ is open.

Extend the language by new function symbols $f_{1}, \ldots, f_{m}$ such that $f_{k}$ is of arity $k$. The symbol $f_{k}$ can be treated as a symbol for a Skolem function for the $k$ th existential quantifier in $\neg \varphi$. Let $\mathcal{T}$ be the set of terms of the 
extended language. We call $\widetilde{\varphi}\left(t_{1}, \ldots, t_{m}\right)$ a Herbrand variant of $\varphi$ if $\widetilde{\varphi}$ is of the form

$$
\bar{\varphi}\left(t_{1}, f_{1}\left(t_{1}\right), \ldots, t_{m}, f_{m}\left(t_{1}, \ldots, t_{m}\right)\right)
$$

for some $t_{1}, \ldots, t_{m} \in \mathcal{T}$.

We say that $\varphi$ is Herbrand provable (in logic) if there is a finite $\mathcal{T}^{\prime} \subseteq \mathcal{T}$ such that

$$
\bigvee_{t_{1}, \ldots, t_{m} \in \mathcal{T}^{\prime}} \widetilde{\varphi}\left(t_{1}, \ldots, t_{m}\right)
$$

is a propositional tautology.

Assume now that $T=\left\{\phi_{1}, \phi_{2}, \ldots\right\}$ is a fragment of arithmetic and + , . are treated as relations. Assume that $\phi_{j}$ is of the form

$$
\forall x_{1} \exists y_{1} \ldots \forall x_{m} \exists y_{m} \bar{\phi}_{j}\left(x_{1}, y_{1}, \ldots, x_{m}, y_{m}\right),
$$

where $\bar{\phi}$ is open. We may assume that $m \leq j$.

We are aiming at formulating Herbrand type consistency of $T$. To this end we need to extend the language by some function symbols $s_{k}^{j}$ such that $s_{k}^{j}$ is of arity $k$. The symbol $s_{k}^{j}$ is a symbol for a Skolem function for the $k$ th existential quantifier in $\phi_{j}$. We have $k \leq j$. Let the language so obtained be denoted by $\widetilde{L}$. Then, by the above definition, a Herbrand variant of $\neg \phi_{j}$ is a formula $\neg \widetilde{\phi}_{j}\left(t_{1}, \ldots, t_{k}\right)$ of the form

$$
\neg \bar{\phi}_{j}\left(t_{1}, s_{1}^{j}\left(t_{1}\right), \ldots, t_{k}, s_{k}^{j}\left(t_{1}, \ldots, t_{k}\right)\right),
$$

where $t_{1}, \ldots, t_{k}$ are terms of $\widetilde{L}$.

Then $T$ is Herbrand inconsistent if a finite disjunction of some Herbrand variants

$$
\neg \widetilde{\phi}_{j}\left(t_{1}, \ldots, t_{k}\right)
$$

is provable in the propositional calculus.

Hence, $T$ is Herbrand consistent if every finite conjunction of some $\widetilde{\phi}_{j}\left(t_{1}, \ldots, t_{k}\right)$ is consistent with the propositional calculus.

To formalize the property " $T$ is Herbrand consistent" in arithmetic we have to encode the language $\widetilde{L}$ in arithmetic. So we number all terms of $\widetilde{L}$ in the following natural order. Let the constants 0,1 be terms of rank 0 and let the terms of rank at most $i+1$ consist of all terms of rank at most $i$ and of all terms of the form $s_{k}^{j}\left(t_{1}, \ldots, t_{k}\right)$ for $j \leq i+1-(k-1)$, with $t_{1}, t_{2}, \ldots, t_{k}$ of rank $i-(k-1), i-(k-2), \ldots, i$ respectively. We number terms of rank 0 , then of rank 1 etc. by consecutive natural numbers leaving some numbers not used. Let the numbers left aside serve to number logical symbols of the language $\widetilde{L}$. The exact form of our numbering is given below in this section. Then terms of rank at most $i$ are numbered by numbers less than $l_{i}$, for some recursive function $i \mapsto l_{i}$. 
As a matter of fact in our numbering every term has a lot of numbers. If $t_{1}, \ldots, t_{k}$ are of rank $i_{0}$ then they are also of rank at most $i$ for every $i \geq i_{0}$, and so the term $s_{k}^{j}\left(t_{1}, \ldots, t_{k}\right)$ is a term of rank at most $i+1$ for every $i \geq i_{0}$.

There are recursive uniformly definable functions $S_{k}^{i, j}$ such that $S_{k}^{i, j}$ : $\left[0, l_{i-(k-1)}\right) \times\left[0, l_{i-(k-2)}\right) \times \ldots \times\left[0, l_{i}\right) \rightarrow\left[0, l_{i+1}\right)$ and the following holds: if the terms $t_{1}, \ldots, t_{k}$ are numbered by $a_{1}, \ldots, a_{k}$ then the term $s_{k}^{j}\left(t_{1}, \ldots, t_{k}\right)$ as a term of rank $i+1$ is numbered by $S_{k}^{i, j}\left(a_{1}, \ldots, a_{k}\right)$.

Let the encoded language be denoted by $L^{*}$. Let $E_{i}$ denote the collection of encoded atomic and negated atomic formulas on terms of rank at most $i$.

We shall call a function $p: E_{i} \rightarrow\{0,1\}$ a T-evaluation of rank $i$ if $p(\neg \varphi)=1-p(\varphi)$ for $\varphi \in E_{i}$. Each such $p$ extends uniquely (in a routine way) to open sentences of $L^{*}$ with terms $<l_{i}$. We assume further that $p(\varphi)=1$ for every axiom of equality $\varphi$ and that $p$ makes

$$
\bar{\phi}_{j}\left(t_{1}, s_{1}^{j}\left(t_{1}\right), \ldots, t_{k}, s_{k}^{j}\left(t_{1}, \ldots, t_{k}\right)\right)
$$

true for every Herbrand variant of $\phi_{j}$ with terms of rank at most $i$, i.e. $p$ takes value 1 at the formula

$$
\bar{\phi}_{j}\left(a_{1}, S_{1}^{i_{1}, j}\left(a_{1}\right), \ldots, a_{k}, S_{k}^{i_{k}, j}\left(a_{1}, \ldots, a_{k}\right)\right)
$$

of $L^{*}$, for $a_{1}<l_{i_{1}}, a_{2}<l_{i_{2}}, \ldots, a_{k}<l_{i_{k}}, j<i_{1}<i_{2}<\ldots<i_{k}<i$.

Note that every $T$-evaluation of rank $i+1$ makes true every conjunction of some $\widetilde{\phi}_{j}\left(t_{1}, \ldots, t_{k}\right)$ with $t_{1}, \ldots, t_{k}$ of rank at most $i-(k-1), i-(k-2), \ldots, i$ respectively.

Thus, $T$ is Herbrand consistent if for every $i$ there is a $T$-evaluation of rank $i$.

To be able to define all the required notions at stage $i$ we need $\exp ^{3} i$ to exist. This is because the numbers $l_{i}$ and $E_{i}$ are roughly of size $\exp ^{2} i$ and any $T$-evaluation of rank $i$ is roughly of size $\exp ^{3} i$.

The whole formalization is available in $I \Delta_{0}+\Omega_{m}$. In particular we have a $\Delta_{1}$ formula $V^{T}(p, i)$ expressing " $p$ is a $T$-evaluation of rank $i$ ". Then we may formulate $\operatorname{Hcons}(T)$ as

$$
\forall i \in \log ^{3} \exists p V^{T}(p, i) .
$$

This may be considered a weak form of Herbrand consistency, but it makes our negative results even stronger.

Here is the exact definition of our coding. Let $M$ be a model of $T_{m}$. Define

$$
\begin{aligned}
l_{0} & =2, \\
l_{i+1} & =l_{i}+(i+1) l_{i}+i l_{i} l_{i-1}+\ldots+l_{i} \ldots l_{0} .
\end{aligned}
$$

We have

$$
l_{i} \leq 2^{2^{i}}
$$


for each $i \in \log ^{3}$. For,

$$
l_{i+1}=l_{i}\left(1+(i+1)+i l_{i-1}+\ldots+l_{i-1} \ldots l_{0}\right)=l_{i}\left(1+(i+1)+l_{i}-l_{i-1}\right)
$$

and hence, assuming (2.2) for a given $i>0$, we obtain

$l_{i+1} \leq 2^{2^{i}}\left(1+(i+1)+2^{2^{i}}-l_{i-1}\right)=\left(2^{2^{i}}\right)^{2}+2^{2^{i}}\left(1+(i+1)-l_{i-1}\right) \leq 2^{2^{i+1}}$ since, obviously, $l_{j} \geq 1+(j+2)$ for each $j \geq 1$.

The graph of the function $l$ (as a function of $i$ ) is definable in $T_{m}$ (with the help of an ordinary technique, see e.g. [WP1] or [HP]), so that the domain of $l$ is an initial segment. From (2.2) it follows that $l$ is defined at least on $\log ^{3}$. An easy estimation shows that

$$
\log ^{m-1} M=\bigcup_{i \in \log ^{m+1} M}\left[l_{i}, l_{i+1}\right)
$$

(where $[a, b)$ is the interval $\{x: a \leq x<b\}$ ), but we do not use this fact. Define also $\left(i, j\right.$ will denote elements of $\log ^{m}$ throughout)

$$
\begin{aligned}
S_{k}^{i, j}\left(a_{1}, \ldots, a_{k}\right)= & l_{i}+(i+1) l_{i}+i l_{i} l_{i-1} \\
& +\ldots+((i+1)-(k-2)) l_{i} \ldots l_{i-(k-2)} \\
& +j l_{i} \ldots l_{i-(k-1)}+\left(a_{1}, \ldots, a_{k}\right)_{i} \quad \text { for } k \geq 2, \\
S_{1}^{i, j}\left(a_{1}\right)= & l_{i}+j l_{i}+\left(a_{1}\right)_{i}
\end{aligned}
$$

for $1 \leq k \leq i, j \leq i$ and $a_{1}<l_{i-(k-1)}, \ldots, a_{k}<l_{i}$ (otherwise set $S_{k}^{i, j}=0$; also let $S_{1}^{0,0}(0)=2$ and $\left.S_{1}^{0,0}(1)=3\right)$. Here $\left(a_{1}, \ldots, a_{k}\right)_{i}$ denotes the position (a number $\left.\leq l_{i} \ldots l_{i-(k-1)}\right)$ of $\left(a_{1}, \ldots, a_{k}\right)$ in the lexicographical ordering of the product

$$
\left[0, l_{i-(k-1)}\right) \times \ldots \times\left[0, l_{i}\right) .
$$

The graph of $S_{k}^{i, j}$ is (uniformly in $i, j, k$ ) definable in $T_{m}$. The values $S_{k}^{i, j}\left(a_{1}, \ldots, a_{k}\right)$ as in $(2.3)$ fill the interval $\left[l_{i}, l_{i+1}\right)$ for each $i \in \log ^{3} M$. Thus, (2.3) constitutes a numbering of $\log M$ (except 0 and 1).

The inner language $L^{*}$, encoded in $T_{m}$ in the usual way, is obtained from the ordinary arithmetical language $L$ (in which addition and multiplication are treated as relations) by adding elements $a \in \log$ as terms (except the $S_{k}^{i, j}\left(a_{1}, \ldots, a_{k}\right)$ 's with $j=0$, which may serve to define other primitive notions and formulas of $\left.L^{*}\right)$.

Let $T$ be a set of sentences of $L^{*}$. An evaluation $p$ on $E_{i}$ is a $T$-evaluation if $p$ satisfies the following condition (denoted briefly by $p \Vdash^{*} \varphi$ ):

(2.4) for each axiom $\varphi$ of $T$ in its prenex form, $\forall x_{1} \exists y_{1} \ldots \forall x_{m} \exists y_{m} \bar{\varphi}$, if $\varphi$ has index $j \geq 1$ (in a fixed enumeration of $T$ ), then for all $i_{1}<\ldots<i_{m}<i\left(\varphi<i_{1}\right)$ and arbitrary $a_{1}<l_{i_{1}}, \ldots, a_{m}<l_{i_{m}}, p$ assumes the value 1 at $\bar{\varphi}\left(a_{1}, S_{1}^{i_{1}, j}\left(a_{1}\right), \ldots, a_{m}, S_{m}^{i_{m}, j}\left(a_{1}, \ldots, a_{m}\right)\right)$. 
It is understood here that all terms occurring in the axioms of $T$ are less than $l_{i}$. Notice that, roughly, we have $p \leq 2^{l_{i}}$ for each evaluation $p$ on $E_{i}$.

The condition (2.4) generalizes in a natural way as follows. For an evaluation $p$ on $E_{i}$ and a sentence $\varphi$ of $L^{*}$ as in (2.1), which contains parameters $\bar{c}$ and is of the form $\psi(\bar{c})$, where $\psi \in \mathbb{N}$ and $\bar{c}<l_{j}$, write $p \Vdash \varphi$ if

$$
\begin{aligned}
\forall i_{1} \in[j+1, i) \forall a_{1}<l_{i_{1}} & \exists b_{1}<l_{i_{1}+1} \ldots \\
& \forall i_{m} \in\left[i_{m-1}+1, i\right) \forall a_{m}<l_{i_{m}} \exists b_{m}<l_{i_{m}+1}
\end{aligned}
$$

such that $p$ is 1 at

$$
\bar{\varphi}\left(a_{1}, b_{1}, \ldots, a_{m}, b_{m}\right) .
$$

For open $\varphi$ we assume that $p \Vdash \varphi$ if $p(\varphi)=1$. Thus, we have $p \Vdash \varphi$ for each standard axiom $\varphi$ of $T$ and each $T$-evaluation $p$.

All quantifiers in the above definition are bounded by $l_{i} \in \log$ (i.e. $\exp \left(l_{i}\right)$ exists). Hence, using the universal formula Sat we can find a $\Delta_{0}$ formula $F$ with an additional parameter $b$ (bounding the unrestricted quantifier in Sat) such that

$$
p \Vdash \varphi \quad \text { iff } \quad F(p, i, \varphi, b)
$$

for every evaluation $p$ on $E_{i}$, standard $\varphi$ with terms $<l_{i}$ and any $b$ such that $b \geq 2^{l_{i}^{\varphi}}$ (cf. Lessan [L] and Theorem 2 of [DP]). It follows that

$$
p \Vdash \varphi \quad \text { iff } \quad \forall b\left(b \geq 2^{l_{i}^{\varphi}} \Rightarrow F(p, i, \varphi, b)\right)
$$

for every evaluation $p$ on $E_{i}$ and a standard sentence $\varphi$ with terms $<l_{i}$.

Assume that $T$ is $\Delta_{0}$ definable in $T_{m}$. We construct a $\Delta_{1}$ formula $V^{T}$ such that

$$
p \text { is a } T \text {-evaluation on } E_{i} \text { iff } V^{T}(p, i)
$$

iff $\forall b\left(b \geq 2^{\omega_{1}\left(l_{i}\right)} \Rightarrow V_{0}^{T}(p, i, b)\right)$ with bounded $V_{0}^{T}$.

Let $M$ be a (non-standard) model of $T_{m}$ and let $i^{\prime}=i+j \in \log ^{3} M$, where $j>\mathbb{N}$. Every $T$-evaluation $p \in M$ on $E_{i+j}^{M}$ determines a model $M(p, i)$ as follows. Put

$$
a={ }_{p} b \equiv p(" a=b ")=1
$$

for $a, b<l_{i+\mathbb{N}}$. Clearly, $={ }_{p}$ is an equivalence relation on the initial segment $\left[0, l_{i+\mathbb{N}}\right)$ of $M$. Let

$$
M(p, i)=\left\{[a]: a<l_{i+\mathbb{N}}\right\}
$$

consist of equivalence classes and define

$$
[a]+[b]=[c] \quad \text { iff } \quad p(" a+b=c ")=1
$$

and similarly for multiplication and ordering. It follows immediately that

$$
M(p, i)=\varphi \quad \text { iff } \quad p(\varphi)=1
$$

for arbitrary open $\varphi$ with parameters $<l_{i+\mathbb{N}}$ ( $a$ is a name for $[a]$ ). 
Also, directly from the above definition, we obtain the following:

$$
\text { If } p \Vdash \varphi\left(c_{1}, \ldots, c_{n}\right) \text {, then } M(p, i) \models \varphi\left(\left[c_{1}\right], \ldots,\left[c_{n}\right]\right)
$$

for arbitrary standard $\varphi$ with parameters $c_{1}, \ldots, c_{n}<l_{i+\mathbb{N}}$. In particular, $M(p, i)$ is a model of $T \cap \mathbb{N}$ for every $T$-evaluation $p$. The converse of (2.7) is in general not true.

Therefore the formula $\mathrm{HCons}_{m}(\varphi)$, expressing the Herbrand consistency of $\varphi$ with $T_{m}$, can be assumed to have the form

$$
\forall i \in \log ^{3} \exists p V^{T_{m}+\varphi}(p, i) .
$$

More precisely, $\operatorname{HCons}_{m}(\varphi)$ looks like

$$
\forall y \forall i \leq y\left[i \leq \log ^{3} y \wedge y \geq 2^{\omega_{1}\left(l_{i}\right)} \Rightarrow \exists p \leq y \quad V_{0}^{T_{m}+\varphi}(p, i, y)\right] .
$$

Finally, $\operatorname{HCons}\left(T_{m}\right)$ is $\operatorname{HCons}_{m}$ ( " $0=0$ ").

3. In order to prove that $\mathrm{HCons}_{m}$ and $\mathrm{HCons}_{m}^{I_{m}}$ have the required properties we need some auxiliary lemmas.

Lemma 3.2 and Corollary 3.3 show that the models $M(p, i)$ are endextensions of the initial segment $\leq^{M} i$ of $M$. Theorem 3.4 is the main step in proving $(*)$ of the introduction. It shows that $M(p, i)$ is a stretching of $M$ in the sense that an element $i$ of $\log ^{m+1} M$ gets an additional exponent in $M(p, i)$ (falls into $\log ^{m+2} M(p, i)$ ). Finally we prove $(*)$ of the introduction.

3.1. Definition. Let $M \models T_{m}$ be given and $i_{0} \in \log ^{3} M$. Let $p$ be a $T_{m}$-evaluation on $E_{i_{0}}$. For $i<i_{0}$ we define a numeral $\underline{i}$ determined by $p$. The sentence $\forall x \exists y(y=x+1)$ is an axiom of $T_{m}$ and we may assume that this is the first axiom in a fixed enumeration of $T_{m}$. It follows that

$$
\forall a<l_{i} \exists b<l_{i+1} p \Vdash(b=a+1)
$$

for all $i<i_{0}$. Hence there exists a sequence $\left\langle c_{i}: i<i_{0}\right\rangle$ of names such that

$$
p \Vdash\left(c_{0}=0\right) \quad \text { and } \quad p \Vdash\left(c_{i+1}=c_{i}+1\right) \text { for all } i<i_{0} .
$$

Let $\underline{i}=c_{i}$ for $i<i_{0}$.

In the next lemma and corollary we shall show that $\underline{i}$ is a name of the $i$ th integer in the models $M(p, j)$ with $j<i_{0}-\mathbb{N}$, in the case where $i_{0}$ is non-standard.

3.2. Lemma. Let $p, i_{0}$ be as before. If, for some name $a, p \Vdash(a \leq \underline{i})$, then there is a $j \leq i$ such that $p \Vdash(a=\underline{j})$. Moreover, (**) $\varphi\left(i_{1}, \ldots, i_{n}\right)$, where $i_{1}, \ldots, i_{n}<i_{0}$, implies $p \Vdash \varphi\left(\underline{i}_{1}, \ldots, \underline{i}_{n}\right)$, for open $\varphi$ all of whose terms are as indicated.

Proof. Induction on $i<i_{0}$. For $i=0$ we have $p \Vdash(a \leq \underline{i})$, whence $p \Vdash(a \leq 0)$. Since the sentence $\forall x(x \leq 0 \Rightarrow x=0)$ can be assumed to be 
the axiom of $T_{m}$, we get $p \Vdash(a=0)$, whence $p \Vdash(a=\underline{0})$. In the inductive step we apply, in a similar way, the axiom

$$
\forall x, y, z(y=z+1 \wedge x \leq y \Rightarrow x=y \vee x \leq z)
$$

to $p \Vdash(a \leq \underline{i+1})$, i.e. to $p \Vdash(a=\underline{i}+1)$, and obtain $p \Vdash(a=\underline{i+1})$ or $p \Vdash(a \leq \underline{i})$. In the latter case we use the inductive assumption to infer $p \Vdash(a=j)$ for some $j \leq i$.

For the second assertion of the lemma we prove first $p \Vdash(\underline{i}+\underline{j}=\underline{i}+\underline{j})$ for all $i, j$ such that $i+j<i_{0}$. We apply induction on $j$. Since $p$ evaluates $\underline{i}+\underline{0}$ as $\underline{i}+0$, the axiom $\forall x(x+0=x)$ yields immediately $p \Vdash(\underline{i}+\underline{0}=\underline{i})$. For the inductive step, notice that $p$ evaluates $\underline{i}+j+1$ as $\underline{i}+j+1$ and hence as $i+j+1$, by the inductive assumption. On the other hand $p \Vdash(i+j+1=$ $\overline{i+j}+1)$, by definition of the numerals, which yields the required result. In $\overline{\text { a similar }}$ way we prove $p \Vdash(\underline{i} \cdot j=i \cdot j)$ for all $i, j$ such that $i, j<i_{0}$, and also $p \Vdash(\underline{i}<j)$ whenever $i \leq \bar{j}$. This shows that $(* *)$ holds for all atomic (and therefore also for all open) sentences $\varphi$, which finishes the proof of the lemma.

We have the following immediate corollary:

3.3. Corollary. Let $M$ be a model of $T_{m}, i_{0} \in \log ^{3} M$ and $p \in M$ a $T_{m}$-evaluation on $E_{i_{0}+j}$, where $j>\mathbb{N}$. Then the initial segment $\leq i_{0}$ of $M$ is isomorphically embeddable into $M\left(p, i_{0}\right)$ as an initial segment. Consequently, if $a_{1}, \ldots, a_{k} \in M, a_{1}, \ldots, a_{k} \leq i_{0}, \varphi\left(x_{1}, \ldots, x_{k}\right)$ is bounded (with + and $\cdot$ treated as relations) and

$$
M \models \varphi\left(a_{1}, \ldots, a_{k}\right),
$$

then $M\left(p, i_{0}\right) \models \varphi\left(\left[\underline{a}_{1}\right], \ldots,\left[\underline{a}_{k}\right]\right)$.

Recall that $I_{m}=\log ^{m-2} M$.

Note that in the presence of $\Omega_{m}$, the segment $\log ^{m+1} M$ is closed under addition. For, we have

$$
\begin{aligned}
\exp ^{m+1}(2 a) & =\exp ^{m}\left(2^{2 a}\right)=\exp ^{m}\left(\left(2^{a}\right)^{2}\right) \\
& =\exp ^{m}\left(\omega_{0}(\exp (a))\right)=\exp ^{m-1}\left(\omega_{1}\left(\exp ^{2}(a)\right)\right) \\
& =\exp ^{m-2}\left(\omega_{2}\left(\exp ^{3}(a)\right)\right)=\ldots=\omega_{m}\left(\exp ^{m+1}(a)\right) .
\end{aligned}
$$

So, if $\exp ^{m+1}(a)$ exists in a model of $I \Delta_{0}+\Omega_{m}$, then (by $\left.\Omega_{m}\right), \omega_{m}\left(\exp ^{m+1}(a)\right)$ exists, and thus $\exp ^{m+1}(2 a)$ exists. To see that $\exp ^{m+1}(a+b)$ exists provided $\exp ^{m+1}(a)$ and $\exp ^{m+1}(b)$ exist, we show that $\exp ^{m+1}(2 \max (a, b))$ exists, and then using the $\Delta_{0}$ minimum principle we infer the existence of $\exp ^{m+1}(a+b)$.

It follows that $I_{m}$ is closed under $\omega_{2}$.

The following theorem implies the property $(*)$ of Section 1. 
3.4. Theorem. Let $M$ be a model of $T_{m}$ and $i_{0} \in \log ^{m+1} M, i_{0}>\mathbb{N}$. Let $p \in M$ be a $T_{m}$-evaluation on $E_{2 i_{0}}$. Then the model $M\left(p, i_{0}\right)$ satisfies

$$
T_{m}+\left[\underline{i}_{0}\right] \in \log ^{m+2} .
$$

Proof. Since $\Omega_{m}$ is an axiom of $T_{m}$ we have $p \Vdash\left(\forall x \exists y y=\omega_{m}(x)\right)$ and so

$$
\forall a<l_{i} \exists b<l_{i+1} p \Vdash\left(b=\omega_{m}(a)\right)
$$

for each $i<i_{0}$. From (2.5) it follows that, for a fixed $\varphi$, the relation $p \Vdash \varphi$ is $\Delta_{0}$ over $M$. Thus, there is a (code of a) sequence $\left\langle w_{i}: i \leq i_{0}\right\rangle \in M$ of names satisfying

$$
\forall i<i_{0} p \Vdash\left(w_{i+1}=\omega_{m}\left(w_{i}\right)\right) \quad \text { and } \quad p \Vdash\left(w_{0}=\exp ^{m} 2\right) .
$$

Clearly there is a standard $n_{0}$ (depending on the position of $\Omega_{m}$ in the enumeration of axioms) such that $w_{i}<l_{i+n_{0}}$ for each $i \leq i_{0}$.

Provably in $T_{m}$, we have

$$
\exp ^{m+2}(k)=\omega_{m}^{k}\left(\exp ^{m} 2\right)
$$

for each $k \in \log ^{m+2}$ (the superscript $k$ denotes the $k$ th iteration). This can be proved in $T_{m}$ by straightforward induction on $l \leq k$ applied to the formula $\exp ^{m+2}(l)=\omega_{m}^{l}\left(\exp ^{m} 2\right)$ which can be bounded by $\omega_{m}\left(\exp ^{m+2}(k)\right)$.

In fact the right hand side of (3.6), i.e. $y=\omega_{m}^{k}\left(\exp ^{m} 2\right)$ can be defined by an arithmetical formula with the help of the Gödel $\beta$-function: let $\psi(x, y, a, b)$ be

$$
\beta(a, b, 0)=\exp ^{m} 2 \wedge \beta(a, b, x)=y \wedge \forall i<x \beta(a, b, i+1)=\omega_{m}(\beta(a, b, i))
$$

where $\beta(a, b, i)=r$ stands for

$$
\exists q(a=q(b(i+1)+1)+r \wedge r<b(i+1)+1) .
$$

Now, $y=\omega_{m}^{x}\left(\exp ^{m} 2\right)$ can be defined by the formula $\exists a, b \psi(x, y, a, b)$.

In order to find a small enough name for a sequence corresponding to the $w_{i} \mathrm{~s}$, let $\mathfrak{M}$ be the model $M\left(p, i_{0}\right)$ determined by $p$ over $M$ and consider the sequence $s$ of iterations

$$
s=\left\langle\exp ^{m} 2, \omega_{m}\left(\exp ^{m} 2\right), \ldots, \omega_{m}^{[\underline{k}]}\left(\exp ^{m} 2\right)\right\rangle
$$

of $\omega_{m}$ in $\mathfrak{M}$, where $[\underline{k}]$ is the maximal $j$ with the property $\omega_{m}^{j}\left(\exp ^{m} 2\right) \leq$ $\left[w_{i_{0}}\right]$ in $\mathfrak{M}$. Since the length and terms of $s$ are relatively small, a standard reasoning shows that $s$ has a $\beta$-code $(a, b)$ in $\mathfrak{M}$, i.e.

$$
\forall i \leq[\underline{k}] \beta(a, b, i)=\omega_{m}^{i}\left(\exp ^{m} 2\right)
$$

in $\mathfrak{M}$. Since $\mathfrak{M}=M\left(p, i_{0}\right)$, the elements $a, b$ have names $A$ and $B$, respectively, with $A, B<l_{i_{0}+n_{1}}$ (for some standard $n_{1} \in \mathbb{N}$ ).

Moreover, there are names $q_{i}, r_{i}<l_{i_{0}+n_{i}}$ for an $n_{i} \in \mathbb{N}$ such that

$$
p \Vdash\left(A=q_{i}(B(i+1)+1)+r_{i} \wedge r_{i}<B(i+1)+1\right),
$$

for each $i \leq k$. 
We shall show that there is a sequence $\left\langle q_{i}, r_{i}: i \leq k\right\rangle$ in $M$ such that $q_{i}, r_{i}<l_{i_{0}+n_{i}}$ for an $n_{i} \in \mathbb{N}$ and (3.7) holds.

For, we have in $M$

$$
\forall i \leq k \exists q_{i}, r_{i} p \Vdash\left(A=q_{i}(B(i+1)+1)+r_{i} \wedge r_{i}<B(i+1)+1\right) .
$$

Choose now $q_{i}, r_{i}$ in $M$, for $i \leq k$, so that $q_{i}, r_{i}$ satisfy (3.7) and the least $j$ such that $q_{i}, r_{i}<l_{i_{0}+j}$ is the least possible $j$ for which suitable $q_{i}, r_{i}$ exist. Then $j \in \mathbb{N}$ and the sequence $\left\langle q_{i}, r_{i}: i \leq k\right\rangle$ is $\Delta_{0}$ definable in $M$, so it is in $M$.

An easy induction in $M$ shows that

$$
p \Vdash\left(r_{i}=w_{i}\right)
$$

for each $i \leq k$. For, assume (3.8) for a given $i<k$. Thus

$$
\mathfrak{M}=\left[r_{i}\right]=\left[w_{i}\right] .
$$

By construction of the $w^{\prime}$ 's, $p \Vdash\left(w_{i+1}=\omega_{m}\left(w_{i}\right)\right)$. Hence $\left[w_{i+1}\right]=\omega_{m}\left(\left[r_{i}\right]\right)$ $=\left[r_{i+1}\right]$ in $\mathfrak{M}$, which proves (3.8).

In particular we have

$$
\left[r_{k}\right]=\left[w_{k}\right] .
$$

Suppose $k<i_{0}$. Then $p \Vdash\left(w_{k+1}=\omega_{m}\left(w_{k}\right)\right)$, whence in $\mathfrak{M}$,

$$
\begin{aligned}
\omega_{m}^{[\underline{k+1}]}\left(\exp ^{m} 2\right) & =\omega_{m}\left(\omega_{m}^{[\underline{k}]}\left(\exp ^{m} 2\right)\right)=\omega_{m}\left(\left[r_{k}\right]\right) \\
& =\omega_{m}\left(\left[w_{k}\right]\right)=\left[w_{k+1}\right] \leq\left[w_{i_{0}}\right],
\end{aligned}
$$

which contradicts the maximality of $k$. Hence $k=i_{0}$, and therefore (3.8) holds for each $i \leq i_{0}$.

Note that

$$
\mathfrak{M}=\left[r_{i}\right]=\omega_{m}^{[\underline{i}]}\left(\exp ^{m} 2\right),
$$

by the choice of $a, b$ and $A, B$. Hence

$$
\mathfrak{M} \models\left[w_{i_{0}}\right]=\omega_{m}^{\left[\underline{i}_{0}\right]}\left(\exp ^{m} 2\right)=\exp ^{m+2}\left[\underline{i}_{0}\right] .
$$

Thus the proof of the theorem is complete.

Now we shall show $(*)$ of Section 1 . Consider first a model $M$ of

$$
\left.T_{m}+\exists \bar{x} \in \log ^{m+1} \varphi(\bar{x})+\operatorname{HCons}^{I_{m}} \text { (" } 0=0 "\right) \text {. }
$$

Let $\bar{a} \in \log ^{m+1} M, \bar{a}=a_{1}, \ldots, a_{k}$, be such that $M \models \varphi(\bar{a})$. Let $i_{0}=$ $\max \bar{a}$. Since $\log ^{m+1} M$ is closed under addition we infer

$$
M \models \exists p V^{T_{m}}\left(p, 2 i_{0}\right) .
$$

Fix $p$. By Corollary 3.3, $M\left(p, i_{0}\right) \models \varphi\left(\left[\underline{a}_{1}\right], \ldots,\left[\underline{a}_{k}\right]\right)$. By Theorem 3.4,

$$
M\left(p, i_{0}\right) \models T_{m}+\varphi\left(\left[\underline{a}_{1}\right], \ldots,\left[\underline{a}_{k}\right]\right)+\left[\underline{a}_{1}\right], \ldots,\left[\underline{a}_{k}\right] \in \log ^{m+2} .
$$

Hence the theory

$$
T_{m}+\exists \bar{x} \in \log ^{m+2} \varphi(\bar{x})
$$

is consistent and $(*)$ follows. 
Added in proof. Recently two new manuscripts on a similar subject have appeared: [W1] — a solution of the original version of the Paris-Wilkie problem, and $[\mathrm{S}]$ - a new partial solution.

\section{References}

[A] Z. Adamowicz, A contribution to the end-extension problem and the $\Pi_{1}$ conservativeness problem, Ann. Pure Appl. Logic 61 (1993), 3-48.

[A1] -, On Tableau consistency in weak theories, circulated manuscript, 1996; preprint 618, Inst. Math., Polish Acad. Sci., 2001.

[AZ] Z. Adamowicz and P. Zbierski, On Herbrand consistency in weak arithmetic, Arch. Math. Logic 40 (2001), 399-413.

[B] S. R. Buss, Bounded Arithmetic, Bibliopolis, 1986.

[DP] C. Dimitracopoulos and J. Paris, Truth definitions for $\Delta_{0}$ formulae, in: Logic and Algorithmic, Monograph. Enseign. Math. 30, Univ. Genève, 1982, 317-329.

[HP] P. Hájek and P. Pudlák, Metamathematics of First-Order Arithmetic, Perspectives in Mathematical Logic, Springer, Berlin, 1993.

[L] H. Lessan, Models of arithmetic, dissertation, Manchester.

$[\mathrm{PW}] \quad$ J. Paris and A. Wilkie, $\Delta_{0}$ sets and induction, in: Open Days in Model Theory (Jadwisin, 1981), W. Guzicki et al. (eds.), Leeds Univ. Press, 1981, 237-248.

[P] P. Pudlák, Cuts, consistency statements and interpretations, J. Symbolic Logic 50 (1985), 423-441.

[S] S. Salehi, Herbrand consistency in arithmetic with bounded induction, $\mathrm{PhD}$ thesis, Inst. Math., Polish Acad. Sci., submitted.

[W] D. Willard, The semantic Tableau version of the second incompleteness theorem extends almost to Robinson's arithmetic $Q$, in: Automated Reasoning with Semantic Tableaux and Related Methods, Lecture Notes in Comput. Sci. 1847, Springer, 2000, 415-430.

[W1] - How to extend the semantic Tableaux and cut-free versions of the second incompleteness theorem almost to Robinson's arithmetic Q, J. Symbolic Logic, to appear.

[WP1] A. Wilkie and J. Paris, On the scheme of induction for bounded arithmetic formulas, Ann. Pure Appl. Logic 35 (1987), 261-302.

[WP2] -, - On the existence of end extensions of models of bounded induction, in: Logic, Methodology and Philosophy of Science, VIII (Moscow, 1987), Stud. Logic Found. Math. 126, North-Holland, 1989, 143-161.

Institute of Mathematics

Polish Academy of Sciences

Śniadeckich 8

00-950 Warszawa, Poland

E-mail: zosiaa@impan.gov.pl

Received 6 November 2000;

in revised form 21 June 2001 